\title{
Effect of the COVID-19 pandemic on acute stroke reperfusion therapy: data from the Lyon Stroke Center Network
}

\section{Cécile PLUMEREAU}

Hospices Civils de Lyon

\section{Tae-Hee $\mathrm{CHO}$}

Hospices Civils de Lyon

\section{Marielle BUISSON}

Hospices Civils de Lyon

\section{Camille AMAZ}

Hospices Civils de Lyon

\section{Matteo CAPPUCCI}

Hospices Civils de Lyon

Laurent DEREX

Hospices Civils de Lyon

\section{Elodie ONG}

Hospices Civils de Lyon Julia FONTAINE

Hospices Civils de Lyon Lucie RASCLE

Hospices Civils de Lyon

Roberto RIVA

Hospices Civils de Lyon

David SCHIAVO

Hospices Civils de Lyon

Axel BENHAMED

Hospices Civils de Lyon

Marion DOUPLAT

Hospices Civils de Lyon

Thomas BONY

Hospices Civils de Lyon

Karim TAZAROURTE

Hospices Civils de Lyon

Célia TUTTLE 
Hospices Civils de Lyon

\section{Omer Frank EKER}

Hospices Civils de Lyon

\section{Yves BERTHEZENE}

Hospices Civils de Lyon

\section{Michel OVIZE}

Hospices Civils de Lyon

\section{Norbert NIGHOGHOSSIAN}

Hospices Civils de Lyon

Laura MECHTOUFF ( $\square$ laura.mechtouff@chu-lyon.fr )

Hospices Civils de Lyon https://orcid.org/0000-0001-9165-5763

\section{Research Article}

Keywords: stroke, thrombolysis, thrombectomy, COVID-19

Posted Date: August 12th, 2020

DOI: https://doi.org/10.21203/rs.3.rs-56553/v1

License: (1) (1) This work is licensed under a Creative Commons Attribution 4.0 International License. Read Full License

Version of Record: A version of this preprint was published at Journal of Neurology on September 9th, 2020. See the published version at https://doi.org/10.1007/s00415-020-10199-6. 


\section{Abstract}

\section{Background}

The coronavirus disease 2019 (COVID-19) pandemic would have particularly affected acute stroke care. However, its impact is clearly inherent to the local stroke network conditions. We aimed to assess the impact of COVID-19 pandemic on acute stroke care in the Lyon comprehensive stroke center during this period.

\section{Methods}

We conducted a prospective data collection of patients with acute ischemic stroke (AIS) treated with intravenous thrombolysis (IVT) and/or mechanical thrombectomy (MT) during the COVID-19 period (from $29 / 02 / 2020$ to 10/05/2020) and a control period (from 29/02/2019 to 10/05/2019). The volume of reperfusion therapies and pre and intra-hospital delays were compared during both periods.

\section{Results}

A total of 208 patients were included. The volume of IVT significantly decreased during the COVID-period (55 (54.5\%) vs $74(69.2 \%) ; p=0.03)$ and was mainly due to time delay among patients treated with MT. The volume of MT remains stable over the two periods (72 (71.3\%) vs $65(60.8 \%)$; $p=0.14)$ but the door-togroin puncture time increased in patients transferred for MT (237 [187-339] vs 210 [163-260]; $p<0.01)$. The daily number of Emergency Medical Dispatch calls considerably increased (1502 [1133-2238] vs 1023 [960-1410]; $p<0.01)$.

\section{Conclusions}

Our study showed a decrease of the volume of IVT, whereas the volume of MT remained stable although intra-hospital delays increased for transferred patients during the COVID-19 pandemic. These results contrast in part with the national surveys and suggest that the impact of the pandemic may depend on local stroke care networks.

\section{Introduction}

The healthcare system has been disrupted during the coronavirus disease 2019 (COVID-19) pandemic outbreak, leading to a massive redistribution of health care resources. The saturation of the Emergency Medical Dispatch (EMD) with COVID 19-related calls may have jeopardized the recognition and management of other emergencies [1]. In addition, this pandemic has imposed containement and social distancing measures, with potential subsequent social isolation that may have contributed to a drop in stroke admissions. Patients' fear of contracting the infection in hospitals may have delayed or limited their demand for care, especially for transient or minor symptoms. Furthermore, the unprecedented media concentration on the pandemic may have precipitated the extinction of calls for other emergencies and insidiously replaced other healthcare needs in the collective mind [2]. 
The effect of the COVID-19 pandemic on stroke care is still debated. Although some studies have reported an impact of the pandemic on acute ischemic stroke (AIS) care in terms of admissions and reperfusion therapies volumes along with longer treatment times and a decrease in the use of stroke imaging compared with control periods in 2019 , other reports have not detected significant effects on revascularization procedures [3-10].

\section{Aims and/or hypothesis}

The objective of our study was to assess the impact of the COVID-19 pandemic on the volume of AIS patients treated with intravenous thrombolysis (IVT) and/or mechanical thrombectomy (MT), as well as pre and intra-hospital delays.

\section{Methods}

\section{1/ Study design and data collection}

Data from these patients were collected within a regional emergency stroke network registry (RESUVAL), approved by the local ethics committee (Comité de Protection des Personnes Sud-Est II, registration E2012-069). This observational study was carried out in accordance with the ethical standards of the Declaration of Helsinki. No patient expressed opposition to the research. The Lyon Stroke Center (tertiary university hospital) serving the greater Lyon metropolitan area (population: 2.3 million), treats $\sim 1,700$ ischemic stroke patients each year and is only comprehensive stroke center (CSC, i.e. thrombectomycapable) within our regional stroke network (5 primary stroke centers, population: 3 million) (Fig. 2).

The analyzed period ranged from the entry into level-2 of the pandemic in France (February $29^{\text {th }}$ ) and the lifting of lockdown on May $10^{\text {th }}$. The same period in 2019 served as control. To take into account the local trend (i.e. yearly increase in case volumes), we also provided data about the total number of reperfusion procedures from January $1^{\text {st }}$ to May $10^{\text {th }}$ of the previous five years.

\section{2/ Study population}

All consecutive patients with AIS treated with IVT and/or MT in the Lyon Stroke Center, France, were included during the COVID-19 period and the control periods.

Baseline data on demographic characteristics, risk factors and medical history were systematically collected at admission as well as times from stroke onset to hospital admission (to our CSC or to primary stroke center or to emergency department as appropriate), door to imaging, door to needle and door to groin puncture. When the time of symptoms onset was unknown, the time when patients woke up or were identified was considered as the time of symptom onset. Neurological status was assessed by board certified neurologists using National Institute of Health Stroke Scale (NIHSS) score at admission.

Data about the daily number of calls to EMD were obtained from the hospital administrative database. 


\section{3/ Imaging}

The first-line imaging method in our CSC is magnetic resonance imaging (MRI), including diffusionweighted imaging (DWI), T2*-weighted imaging, Fluid-Attenuated-Inversion-Recovery (FLAIR), 3D-Timeof-Flight MR-angiography (MRA), perfusion-weighted imaging and cervical-vessels angiography were optional. If MRI was unavailable or contra-indicated, non-enhanced computed tomography (CT) followed by CT-angiography were performed; CT- perfusion was optional. Lesion side and baseline ischemic core size were assessed on DWI or CT using the Alberta Stroke Program Early CT Score (ASPECTS) for patients with stroke in the middle cerebral artery territory [11]. Baseline arterial occlusion site was evaluated with MRA or CT-angiography. A follow-up CT performed at 24 hours classified any hemorrhagic transformation according to the European Co-operative Acute Stroke Study (ECASS II) classification [12].

\section{4/ Statistical analysis}

Continuous variables are expressed as means (standard deviation [SD]) or medians (interquartile range $[\mathrm{IQR}])$, and categorical variables as percentages. The Mann-Whitney U-test and Fisher's exact test were used to compare continuous and categorical variables, respectively. A p-value $<0.05$ was considered significant. Data were analyzed with Stata Version $15^{\mathrm{TM}}$ (STATACORP, COLLEGE STATION, TEXAS 77845 USA).

\section{Results}

A total of 208 patients were included in this study, 107 during the COVID-19 and control periods, respectively. Patients' main characteristics are shown in Table 1. Current smoking (18 (17.8\%) vs 33 $(30.8 \%) ; p=0.04)$ and posterior circulation stroke (3 (3.0) vs $15(14.0) ; p<0.01)$ were less frequent during the COVID-19 period. Seven patients (6.5\%) had typical CT findings and/or PCR confirmed COVID-19 infection.

The volume of IVT was significantly lower during the COVID-19 period compared to the control period (55 $(54.5 \%)$ vs $74(69.2 \%) ; p=0.03)$. The main factor for limiting the use of IVT among patients treated with MT was time delay (patients admitted beyond $270 \mathrm{~min}$ from symptoms onset: 25 (58.1\%) vs $9(28.1 \%)$ in the COVID-19 and control periods, respectively; $p<0.01)$. In contrast, the volume of patients treated with MT remained stable over these two periods (72 (71.3\%) during the COVID-19 period vs $65(60.8 \%)$ during the control period; $p=0.14)$. The number of revascularization procedures in Lyon CSC steeply and yearly increased since 2015 (Fig. 2). The curve of cumulative cases in early 2020 was superior to that of 2019, but leveled off at the end of February 2020, and thereafter remained inferior to 2019 levels.

Onset-to-door, door-to-imaging and door-to-needle times did not differ between the two periods. In contrast, door-to-groin puncture time was increased in patients transferred for MT (237 [187-339] vs 210 [163-260]; $p<0.01$ ) (Table 2). Note that the volume of direct admissions to CSC declined without reaching the statistical significance threshold. 
The emergency call center faced a significant increase in activity during the COVID-19 period. The total daily number of calls increased considerably (1023 [960-1410] vs 1502 [1133-2238] in the control and COVID-19 periods, respectively; $p<0.01$ ).

\section{Discussion}

We evaluated the impact of COVID-19 outbreak on a regional stroke care network. We observed a decrease of the volume of IVT, whereas the volume of MT remained stable although we observed prolonged intra-hospital time for transferred patients.

In line with previous studies, the volume of IVT markedly decreased during the COVID-19 period [3-5, 7-10]. The volume of MT remained stable between the 2 periods while previous studies have reported conflicting results [3-5, 8-10]. The regional variability of the impact of COVID-19 on acute stroke care is illustrated by a German study which found a relevant effect on MT in a only one out of four centers [10]. Our result must be interpreted in relation to the resources available for stroke care delivery in our catchment area, which is likely undersized with regard to the large population base. This discrepancy between supply and demand may have propped up the number of revascularization procedures, despite a likely COVID-related reduction in healthcare resources, including stroke care. Nevertheless, we failed to observe our expected yearly growth in the number of reperfusion procedures. A similar observation was made by Hsiao, et al [8]. The magnitude of the COVID-19 pandemics was also lower in our region compared to other French regions as the Grand-Est, and could have modified its impact of the COVID-19 on stroke care as reported in Germany [10].

Although the volume of MT stayed stable, intra-hospital times, reflected by the door-to-groin puncture time, was delayed for patients transferred for MT as previously reported in a French multicentric study [5]. Our study showed an increase of about 50\% in the total daily number of EMD calls during the COVID-19 period. EMD saturation may explain the increased transfer times: However, this factor does not seem to have an impact on prehospital time since we did not observe any onset-to-door time, increase during the COVID-19 period. Nevertheless, time delay was the most frequent reason for non-thrombolysis among patients treated exclusively with MT.

Another interesting finding is the significant decrease in patients managed for posterior circulation stroke during the pandemic period. Outside the pandemic period, posterior circulation strokes are more likely misdiagnosed in part because of nonspecific clinical presentation [13]. This phenomenon may have been exacerbated during the COVID-19 period. In contrast to other studies, age, NIHSS and ASPECT scores did not differ between the two periods, suggesting that criteria for treatment eligibility remained unchanged $[5,6]$.

Our methodological strengths come from a prospective data collection concerning all consecutive patients treated with MT in our geographical area as our stroke center is the only one to have thrombectomy facilities within our stroke regional network. 
Our study has some limitations. The sample size is limited as a result of the short study period. No data about untreated patients was available in our registry. Still, we prospectively collected data concerning all consecutive patients treated with MT; as our CSC is the only thrombectomy-capable hospital within our stroke regional network, the count of MT cases was exhaustive. The generalizability of these results found in our stroke regional network to other regions or countries with a different stroke care organization is uncertain.

\section{Declarations}

Acknowledgments: We acknowledge Magali Bischoff and Carlos El Khoury from the RESUVAL network, Serkan Cakmak, Karine Blanc-Lasserre, Anne-Evelyne Vallet, Frédéric Philippeau and Chérif Heroum from primary stroke centers.

Funding: Hospices Civils de Lyon Funding

Conflict(s)-of-Interest/Disclosure(s): The authors declare that they have no conflict of interest.

Ethical standards: This research was conducted according to the ethical standards issued by the Declaration of Helsinki.

\section{Consent: Patients were informed and no patient expressed opposition to the research.}

\section{References}

1. the COVID19 IRCCS San Matteo Pavia Task Force, Perlini S, Canevari F, et al (2020) Emergency Department and Out-of-Hospital Emergency System (112-AREU 118) integrated response to Coronavirus Disease 2019 in a Northern Italy centre. Intern Emerg Med. https://doi.org/10.1007/s11739-020-02390-4

2. De Coninck D, d'Haenens L, Matthijs K (2020) Forgotten key players in public health: news media as agents of information and persuasion during the COVID-19 pandemic. Public Health 183:65-66. https://doi.org/10.1016/j.puhe.2020.05.011

3. Zhao J, Li H, Kung D, et al (2020) Impact of the COVID-19 Epidemic on Stroke Care and Potential Solutions. Stroke 51:1996-2001. https://doi.org/10.1161/STROKEAHA.120.030225

4. Baracchini C, Pieroni A, Viaro F, et al (2020) Acute stroke management pathway during Coronavirus19 pandemic. Neurol Sci 41:1003-1005. https://doi.org/10.1007/s10072-020-04375-9

5. Kerleroux B, Fabacher T, Bricout N, et al (2020) Mechanical Thrombectomy for Acute Ischemic Stroke Amid the COVID-19 Outbreak: Decreased Activity, and Increased Care Delays. Stroke 51:2012-2017. https://doi.org/10.1161/STROKEAHA.120.030373

6. Rudilosso S, Laredo C, Vera V, et al (2020) Acute Stroke Care Is at Risk in the Era of COVID-19: Experience at a Comprehensive Stroke Center in Barcelona. Stroke 51:1991-1995. https://doi.org/10.1161/STROKEAHA.120.030329 
7. Pop R, Quenardelle V, Hasiu A, et al (2020) Impact of the COVID-19 outbreak on acute stroke pathways - insights from the Alsace region in France. European Journal of Neurology n/a: https://doi.org/10.1111/ene.14316

8. Hsiao J, Sayles E, Antzoulatos E, et al (2020) Effect of COVID-19 on Emergent Stroke Care: A Regional Experience. Stroke. https://doi.org/10.1161/STROKEAHA.120.030499

9. on behalf of STROKE TEAM Collaborators, Frisullo G, Brunetti V, et al (2020) Effect of lockdown on the management of ischemic stroke: an Italian experience from a COVID hospital. Neurol Sci. https://doi.org/10.1007/s10072-020-04545-9

10. Hoyer C, Ebert A, Huttner HB, et al (2020) Acute Stroke in Times of the COVID-19 Pandemic: A Multicenter Study. Stroke 51:2224-2227. https://doi.org/10.1161/STROKEAHA.120.030395

11. Barber PA, Demchuk AM, Zhang J, Buchan AM (2000) Validity and reliability of a quantitative computed tomography score in predicting outcome of hyperacute stroke before thrombolytic therapy. The Lancet 355:1670-1674. https://doi.org/10.1016/S0140-6736(00)02237-6

12. Hacke W, Kaste M, Fieschi C, et al (1998) Randomised double-blind placebo-controlled trial of thrombolytic therapy with intravenous alteplase in acute ischaemic stroke (ECASS II). The Lancet 352:1245-1251. https://doi.org/10.1016/S0140-6736(98)08020-9

13. Gurley KL, Edlow JA (2019) Avoiding Misdiagnosis in Patients With Posterior Circulation Ischemia: A Narrative Review. Acad Emerg Med 26:1273-1284. https://doi.org/10.1111/acem.13830

\section{Tables}

Table 1: Main characteristics of the study population 


\begin{tabular}{|c|c|c|c|}
\hline & $\begin{array}{c}\text { Control period } \\
(\mathrm{n}=107)\end{array}$ & $\begin{array}{c}\text { COVID-19 period } \\
(\mathrm{n}=101)\end{array}$ & $\begin{array}{c}\mathrm{p}- \\
\text { value }\end{array}$ \\
\hline Age, years & $72.4+/-14.8$ & $70.2+/-14.7$ & 0.24 \\
\hline Male & $49(45.8)$ & $52(51.5)$ & 0.49 \\
\hline prestroke mRS $>2$ & $11(10.8)$ & $5(5.2)$ & 0.20 \\
\hline Hypertension & $60(5.1)$ & $57(56.5)$ & 1 \\
\hline Hyperlipemia & $27(25.2)$ & $29(28.7)$ & 0.64 \\
\hline Diabetes & $18(16.8)$ & $9(8.9)$ & 0.10 \\
\hline Current smoking & $33(30.8)$ & $18(17.8)$ & 0.04 \\
\hline Previous stroke/TIA & $17(15.9)$ & $13(12.9)$ & 0.56 \\
\hline Ischemic heart disease & $16(15.0)$ & $11(11.0)$ & 0.42 \\
\hline Atrial fibrillation & $24(22.4)$ & $13(12.9)$ & 0.10 \\
\hline Antithrombotic drug & $42(39.3)$ & $37(36.6)$ & 0.78 \\
\hline Etiology & & & 0.57 \\
\hline Cardioembolism & $49(46.7)$ & $36(37.1)$ & \\
\hline \multicolumn{4}{|l|}{ atherosclerosis } \\
\hline Microangiopathy & $3(2.9)$ & $5(5.2)$ & \\
\hline Others & $5(4.8)$ & $5(5.2)$ & \\
\hline Undetermined & $24(22.9)$ & $30(30.9)$ & \\
\hline Baseline NIHSS score & $15(5-19)$ & $13(6-18)$ & 0.56 \\
\hline Wake-up stroke & $27(25.2)$ & 32 (31.7) & 0.36 \\
\hline Off-hour & $52(48.6)$ & $55(56.7)$ & 0.26 \\
\hline $\mathrm{CT}$ & $33(30.8)$ & $33(32.7)$ & 0.88 \\
\hline MRI & $83(77.6)$ & $82(81.2)$ & 0.61 \\
\hline \multicolumn{4}{|l|}{ Thrombus location } \\
\hline M1 MCA segment & $56(52.3)$ & $53(52.5)$ & 1 \\
\hline M2 MCA segment & $10(9.4)$ & $11(9.9)$ & 1 \\
\hline Intracranial ICA & $24(22.4)$ & $16(15.8)$ & 0.29 \\
\hline Tandem occlusion & $21(19.6)$ & $14(15.6)$ & 0.58 \\
\hline Posterior circulation & $15(14.0)$ & $3(3.0)$ & $<0.01$ \\
\hline ASPECT score & 8 [7-9] & 8 [7-9] & 0.47 \\
\hline Right hemisphere & $56(52.3)$ & $42(41.6)$ & 0.20 \\
\hline Thrombolysis & $74(69.2)$ & $55(54.5)$ & 0.03 \\
\hline Telethrombolysis & 17 (15.9) & $10(9.9)$ & 0.22 \\
\hline Mechanical thrombectomy & $65(60.8)$ & $72(71.3)$ & 0.14 \\
\hline Secondary transfers & $34(52.3)$ & $44(61.1)$ & 0.31 \\
\hline Reperfusion TICI 2b/3 & 51 (79.7) & $48(67.6)$ & 0.12 \\
\hline Any hemorrhagic & $25(25.8)$ & $29(30.9)$ & 0.52 \\
\hline
\end{tabular}


transformation

Emergency Medical Dispatch

Number of calls / day

$1023[960-1410]$

$1502[1133-2238]$

$<0.01$

mRS : modified Rankin Scale, TIA : transient ischemic accident, NIHSS : National Institute of Health Stroke Score, CT : Computed Tomography, MRI : Magnetic Resonance Imaging, MCA : Middle Cerebral Artery, ICA : internal carotid artery, ASPECT : Alberta Stroke Program Early CT, TICI : Thrombolysis in cerebral Infarction

Table 2: Time intervals

\begin{tabular}{|c|c|c|c|}
\hline & $\begin{array}{l}\text { Control period } \\
\quad(n=107)\end{array}$ & $\begin{array}{l}\text { COVID-19 period } \\
\qquad(\mathrm{n}=101)\end{array}$ & $\begin{array}{c}\text { p- } \\
\text { value }\end{array}$ \\
\hline Onset-to-door time, min & $83[57-117]$ & 88 [59-139] & 0.34 \\
\hline Door-to-imaging time, min & $21(14-40)$ & $22(15-41)$ & 0.59 \\
\hline Door-to-needle time, min & $55(43-80)$ & $55(43-75)$ & 0.88 \\
\hline Door-to-groin puncture time, min & $121[80-210]$ & $185[103-270]$ & 0.01 \\
\hline Primary admission to Lyon & $84[67-94]$ & $81[65-111]$ & 0.68 \\
\hline \multicolumn{4}{|l|}{ CSC } \\
\hline Transfer to Lyon CSC & $210[163-260]$ & 237 [187-339] & 0.04 \\
\hline
\end{tabular}

CSC : comprehensive stroke center

Figures 


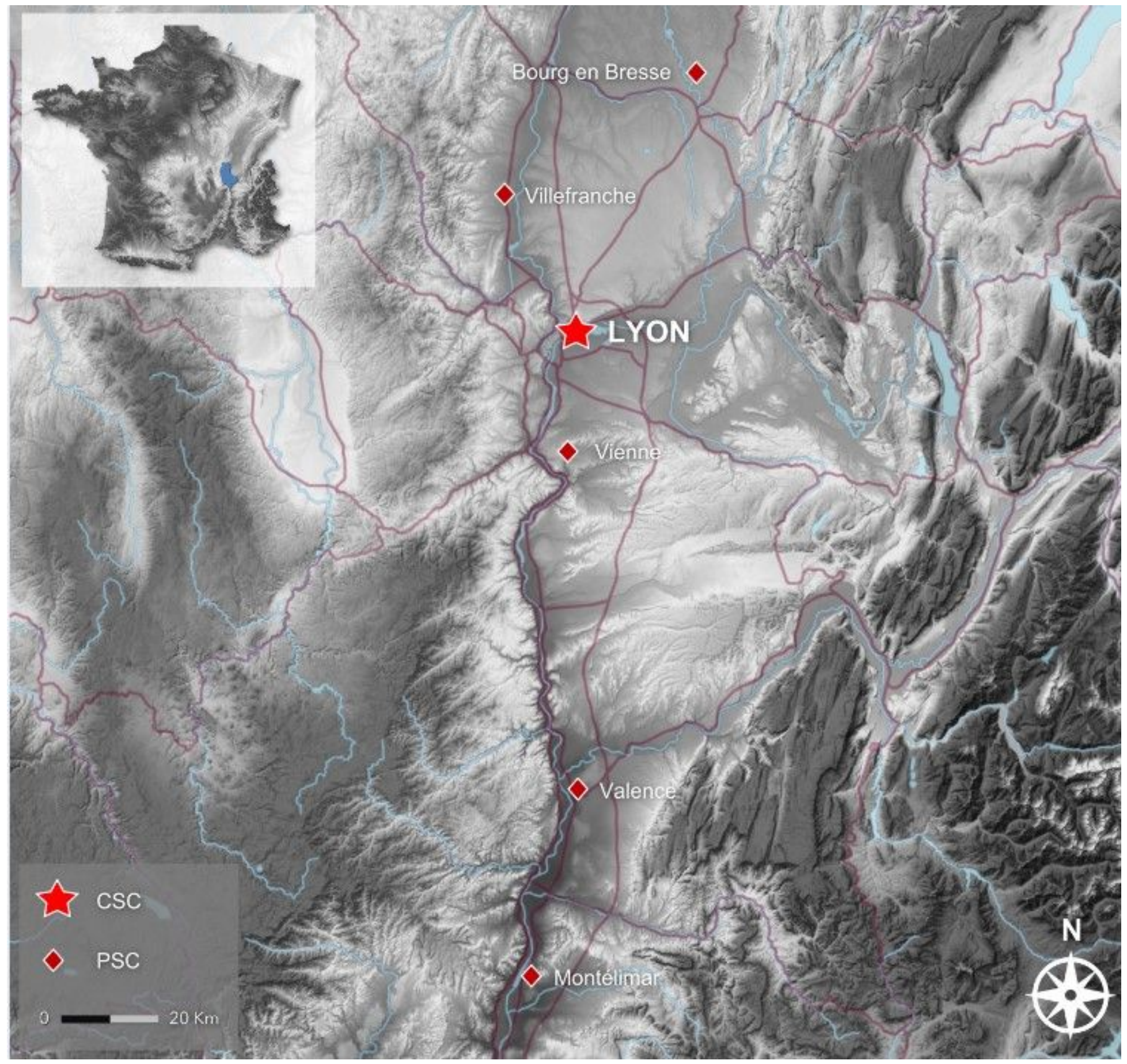

\section{Figure 1}

Stroke care network in the northern Rhône valley (inset, location within France; CSC, Comprehensive Stroke Center; PSC, Primary Stroke Center. Source: Institut national de l'information géographique et forestière (IGN)) 


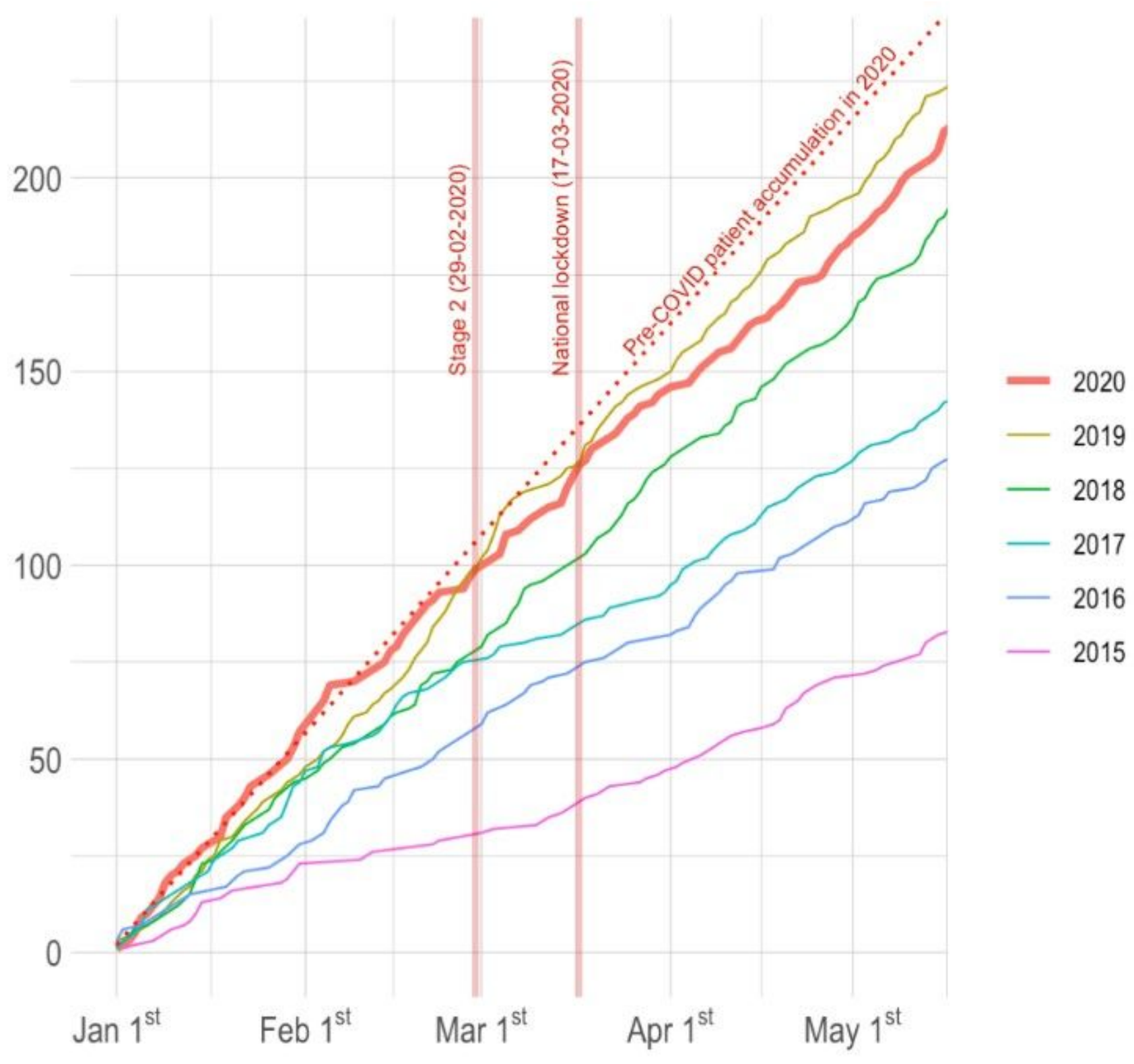

Figure 2

Cumulative number of reperfusion procedures (intravenous thrombolysis and thrombectomy) from January 1st to May 10th from 2015 to 2020 (the dashed line shows the slope of case numbers in 2020 before the COVID-19 period). 